\title{
Enhanced Dispersion Tolerance of Coherent Offset-QAM OFDM over Conventional OFDM
}

\author{
Jian Zhao ${ }^{1}$ and A.D. Ellis ${ }^{2}$ \\ ${ }^{l}$ Photonic Systems Group, Tyndall National Institute and University College Cork, Lee Maltings, Dyke Parade, Cork, Ireland \\ ${ }^{2}$ Aston Institute of Photonic Technology, Aston University, Birmingham B4 7ET, UK \\ Tel: +353214904128,Email: jian.zhao@tyndall.ie
}

\begin{abstract}
We experimentally demonstrate 38-Gbit/s offset-16QAM OFDM over 840km without guard interval, and numerically show that 112-Gbit/s PDM offset-QPSK OFDM achieves 23\% increase in net capacity over conventional OFDM under the same transmission reach. (C) 2014 Optical Society of America

OCIS codes: (060.2330) Fiber optics communications; (060.4080) Modulation
\end{abstract}

\section{Introduction}

Multicarrier techniques have attracted much interest for high-speed optical communication systems. Conventional orthogonal frequency division multiplexing (C-OFDM) [1-3] utilizes a sinc-function spectrum to achieve subchannel orthogonality. By inserting a guard interval (GI) whose length is longer than the memory length of the channel, inter-symbol interference (ISI) and inter-carrier interference (ICI) can be eliminated, and dispersion compensation can be realized using simple one-tap equalizers after subcarrier demultiplexing. However, the sinc function has a long oscillating tail in the frequency domain, resulting in vulnerability to residual ICI and ISI.

Recently, a novel multicarrier scheme, offset quadrature amplitude modulation (offset-QAM) OFDM, was proposed for optical communications [4-6]. This scheme can greatly relax the required signal spectra for subchannel orthogonality [4]. For example, the square-root-raised-cosine (SRRC) function cannot achieve sub-channel orthogonality unless offset-QAM formats are used. In the electronic domain, the concept can be implemented using filter-bank [5] or discrete-Fourier-transform (DFT) [6] based implementations, the latter of which is more computationally efficient and can achieve channel equalization using one-tap equalizers similar to C-OFDM.

In this paper, we investigate chromatic dispersion (CD) tolerance of DFT-based offset-QAM OFDM, and show that the required GI length to enable ISI and ICI free operation in the presence of dispersion scales with twice the subcarrier spacing, rather than the full OFDM bandwidth. Consequently, this scheme may eliminate the needs of GI for dispersion compensation and is more suitable than C-OFDM for long-distance transmissions.

\section{Enhanced tolerance to dispersion without GI}
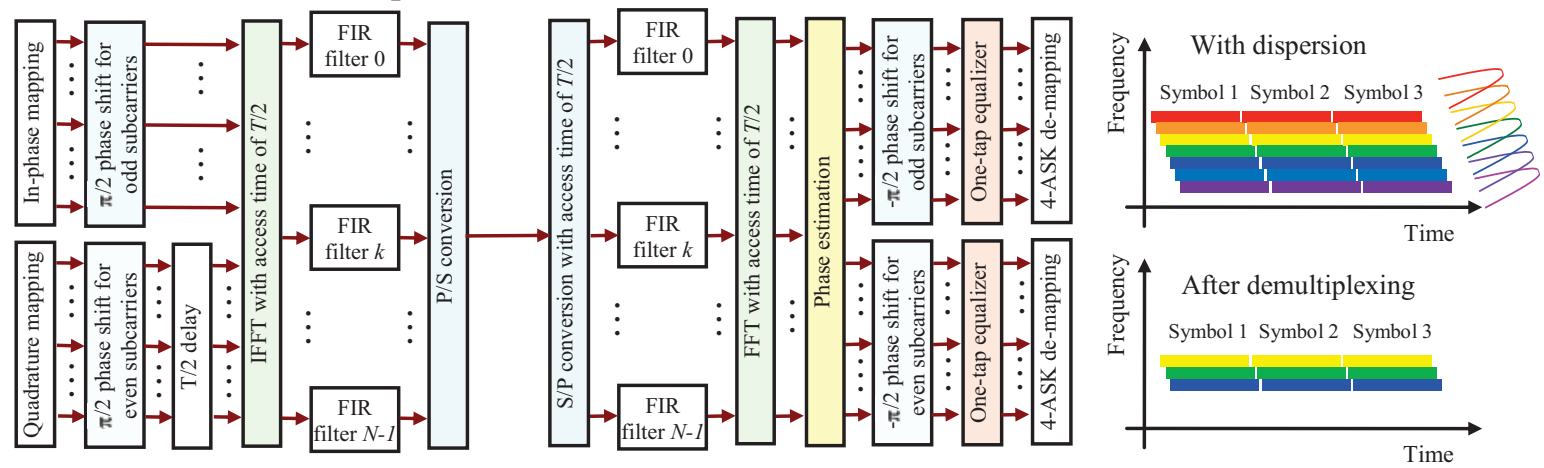

Fig. 1. Coding and decoding of DFT-based offset-QAM OFDM (left) and residual ICI/ISI from adjacent sub-channels without GI (right)

Fig. 1 shows the coding and decoding of DFT-based offset-QAM OFDM. The in-phase and quadrature tributaries are encoded separately. For the in-phase tributary, the phases of even subcarriers are set to be 0 while those of odd subcarriers are set to be $\pi / 2$. For the quadrature tributary, the phases of subcarriers are set conversely. The quadrature tributary is then delayed by $T / 2$ with respect to the in-phase tributary. An inverse fast Fourier transform (IFFT) is applied to generate time-domain samples from the in-phase tributary at times $k T$, and from the quadrature tributary at times $(k+1 / 2) T$, where $k$ is an integer. The generated parallel outputs pass through finite impulse response (FIR) filters for pulse shaping before parallel-to-serial (P/S) conversion. At the receiver, the received signal is serial-to-parallel (S/P) converted with the access time of $T / 2$. The outputs pass through FIR filters that are matched to those at the transmitter. An FFT is applied to transform the signals to the frequency domain. The pilot tone in the subcarriers is extracted for phase estimation. The phase shifts applied to the different subcarriers at the transmitters are reversed in order to obtain the correct data. The signals are equalized using one-tap equalizers. 
The right figures in Fig. 1 illustrate the principle of enhanced tolerance to dispersion in offset-QAM OFDM. In C-OFDM, due to the long spectral tail of the sinc function, all sub-channels would introduce ICI to the target subchannel when the GI is not employed. In order to avoid the ICI and ISI, the length of the GI should be larger than the time delay between the sub-channels that have the largest distance in frequency. In contrast, in offset-QAM OFDM, the spectral tail can be greatly suppressed and the target sub-channel can be demultiplexed without any ISI and ICI from sub-channels with more than sub-channel distance. The required GI length scales with twice the subcarrier spacing in offset-QAM OFDM rather than the full OFDM bandwidth. Consequently, offset-QAM OFDM may potentially support dispersion compensation without any GI for a larger number of subcarriers.

\section{38-Gbit/s offset-16QAM OFDM experiments}

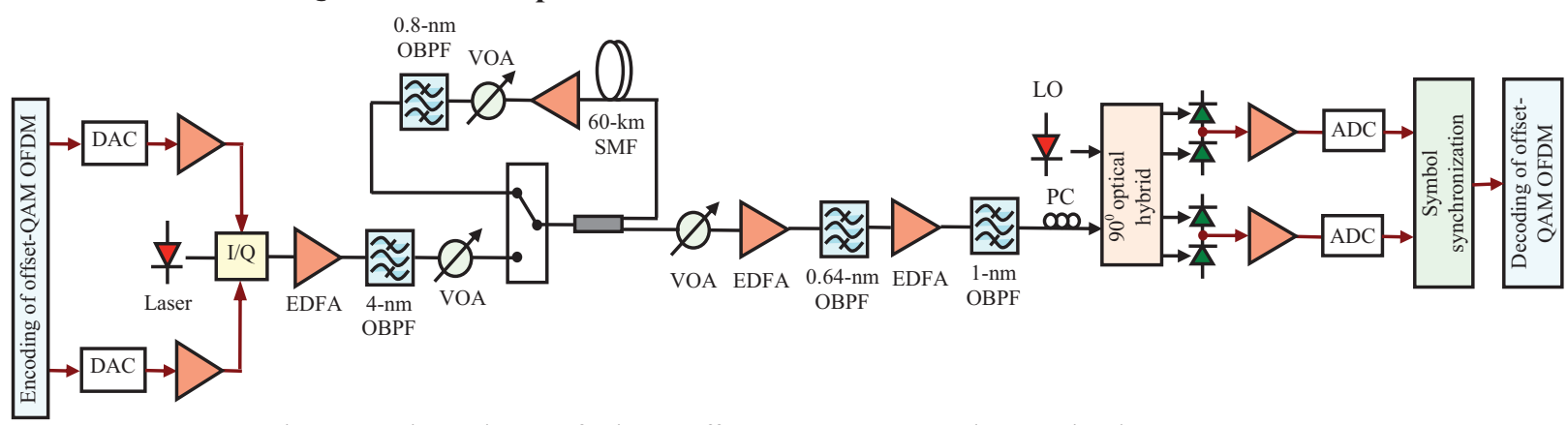

Fig. 2. Experimental setup of coherent offset-16QAM OFDM and conventional 16QAM OFDM

Figure 2 shows the experimental setup. The IFFT and FFT used 128 points, of which 102 subcarriers were used for 16QAM data modulation. The six subcarriers in the zero-frequency region were not modulated, allowing for ACcoupled amplifiers and insertion of pilot tones for phase estimation. The twenty subcarriers in the high-frequency region were zero-padded to avoid aliasing. In offset-QAM OFDM, the FIR filter created a set of SRRC functions with a roll-off coefficient of 0.5 and the $3-\mathrm{dB}$ bandwidth was the same as the subcarrier spacing. The generated signal was downloaded to $12-\mathrm{GSa} / \mathrm{s}$ digital-to-analogue converters (DACs). A laser with 6-kHz linewidth was used to generate the optical carrier. The OFDM signal was fed into an optical I/Q modulator with a peak-to-peak driving swing of $0.5 V_{\pi}$. The generated optical signal was amplified by an erbium doped fiber amplifier (EDFA), filtered by a 4-nm optical band-pass filter (OBPF), and transmitted over a recirculating loop comprising 60-km single-mode fiber (SMF) with 14-dB fiber loss. The noise figure of the EDFA was $6 \mathrm{~dB}$ and another 0.8 -nm OBPF was used in the loop to suppress the amplified spontaneous emission noise. The launch power per span was around $-7 \mathrm{dBm}$ to avoid nonlinear effects. At the receiver, a variable optical attenuator (VOA) was used to vary the optical signal-tonoise ratio (OSNR) for the bit error rate (BER) measurements. The pre-amplifier was followed by an OBPF with a 3-dB bandwidth of $0.64 \mathrm{~nm}$, a second EDFA, and another OBPF with a 3-dB bandwidth of $1 \mathrm{~nm}$. A polarization controller (PC) was used to align the polarization of the OFDM signal before entering the signal path of a $90^{\circ}$ optical hybrid. The optical outputs of the hybrid were connected to two balanced photodiodes, amplified by $40-\mathrm{GHz}$ electrical amplifiers, and captured using a 50-GSa/s real-time oscilloscope. The receiver algorithms included symbol synchronization and decoding (Fig. 1). The total number of measured 16QAM symbols was 240,000.

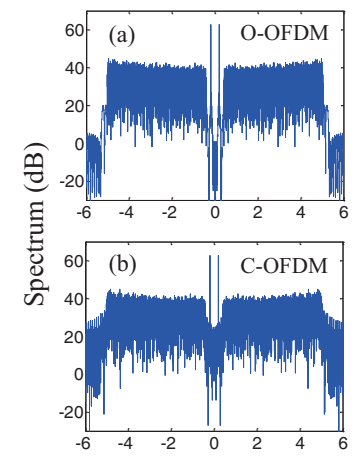

Frequency $(\mathrm{GHz})$
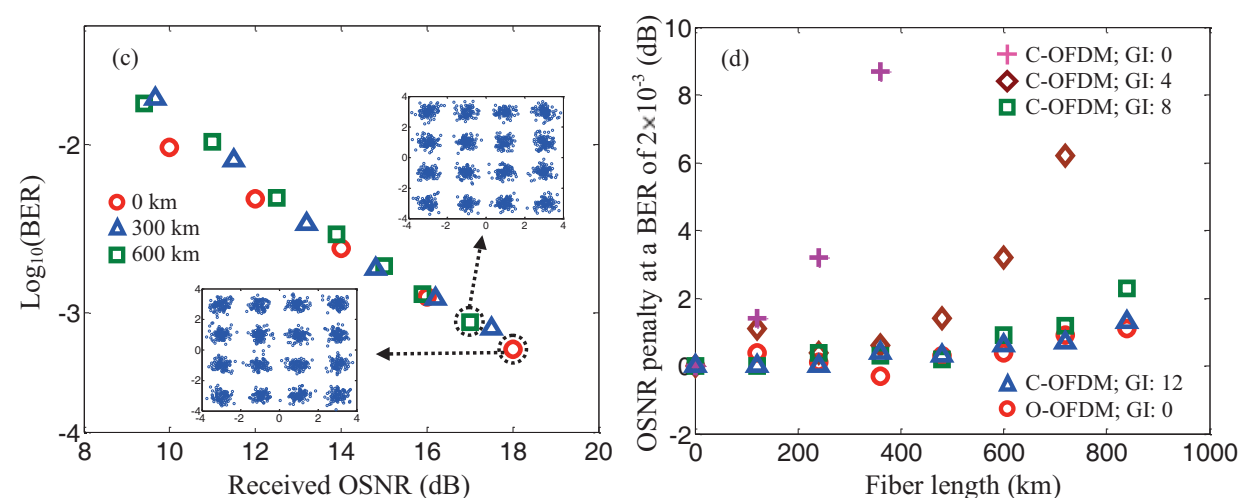

Fig. 3. (a)\&(b) Spectra of offset-16QAM OFDM and conventional 16QAM OFDM; (c) Performance versus the received OSNR for offset16QAM OFDM without GI. Insets are the recovered constellation diagrams at 0 and $600 \mathrm{~km}$. (d) OSNR penalty versus the transmission distance for offset-16QAM OFDM without GI and conventional 16QAM OFDM with different GI lengths. 
Fig. 3(a) and (b) show the spectra of offset-16QAM OFDM and 16-QAM C-OFDM, respectively. Offset-QAM OFDM avoided the long spectral tails, and so exhibited greatly suppressed side lobes. Fig. 3(c) shows BER versus the received OSNR for offset-QAM OFDM after 0-, 300-, and 600-km transmission without GI. Insets illustrate the recovered eye diagrams. It can be seen that offset-QAM OFDM without any GI exhibited negligible penalty after $300-$ and $600-\mathrm{km}$ transmission. This is in contrast to C-OFDM where the requirement for the GI length is much stricter. Figure 3(d) shows OSNR penalty at a BER of $2 \times 10^{-3}$ versus fiber length for offset-QAM OFDM without GI and C-OFDM with different lengths of GI. It is observed that in C-OFDM, when the GI length was zero, the system exhibited a large OSNR penalty of $>8 \mathrm{~dB}$ even at $360 \mathrm{~km}$. A GI length of twelve was required to support $840 \mathrm{~km}$ with similar performance as offset-QAM OFDM without GI. This confirms the advantage of offset-QAM OFDM over C-OFDM in reducing the GI-induced overhead for long-distance fiber transmissions.

\section{112-Gbit/s polarization-division-multiplexed offset-QPSK OFDM simulations}
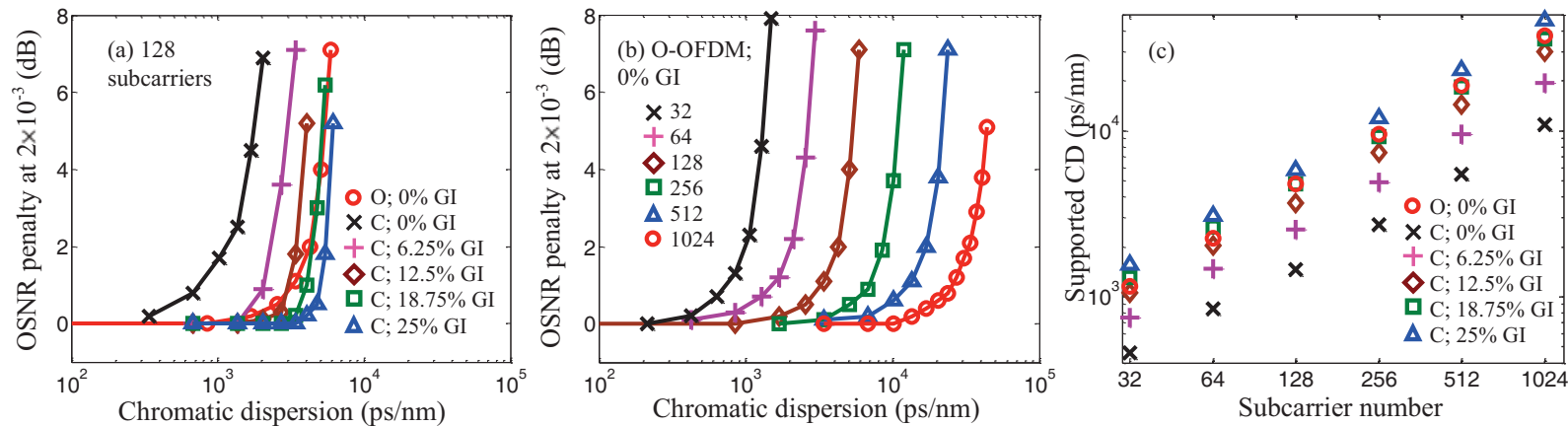

Fig. 4. (a) OSNR penalty versus CD for PDM offset-QPSK OFDM without GI and conventional PDM QPSK OFDM with different GI lengths. The subcarrier number is 128; (b) OSNR penalty versus CD for PDM offset-QPSK OFDM without GI and with different subcarrier numbers; (c) Supported CD values at the 3-dB OSNR penalty versus the subcarrier number for offset-QPSK OFDM and conventional QPSK OFDM.

Polarization-division-multiplexed (PDM) offset-QPSK OFDM and QPSK C-OFDM were simulated to further investigate the enhanced dispersion tolerance. The sampling rates of the DACs and the oscilloscope were $40 \mathrm{GSa} / \mathrm{s}$. The subcarrier number varied from 32 to 1024, and for each case, the signal line rate (including the GI in C-OFDM) was fixed to be $112 \mathrm{Gbit} / \mathrm{s}$. Nonlinearity in the fibers was neglected in the simulations in order to isolate the effects of dispersion. In offset-QAM OFDM, the FIR filter created a set of SRRC functions with a roll-off coefficient of 0.5. Fig. 4(a) shows the OSNR penalty versus CD when the subcarrier number is 128. In C-OFDM, the transmission reach was extended by using a longer GI at the expense of reduced net data rate. On the other hand, at the 3-dB penalty, offset-QPSK OFDM without GI could realize the transmission reach the same as that of the COFDM with $18.75 \%$ GI length. Fig. 4(b) shows the OSNR penalty versus CD for offset-QPSK OFDM with different subcarrier numbers. As expected, the CD tolerance scales with twice the subcarrier spacing, so inversely with the subcarrier number at a fixed OFDM bandwidth. From the figure, it is seen that for 1024 subcarriers, offsetQPSK OFDM could support $\sim 36,000 \mathrm{ps} / \mathrm{nm}$ without any GI at the 3-dB penalty. Fig. 4(c) illustrates supported CD values at the $3-\mathrm{dB}$ penalty versus the subcarrier number. It can be seen that the transmission reaches scaled linearly with the subcarrier number for both offset-QPSK OFDM and C-OFDM. Regardless of the subcarrier number, $18.75 \%$ GI was required in C-OFDM to achieve the same performance as offset-QPSK OFDM without any GI. Therefore, the presented scheme is more suitable for long-distance transmissions, with $23 \%$ increase in net data rate.

\section{Conclusion}

We have experimentally shown that 38-Gbit/s offset-16QAM OFDM can support 840-km transmission with negligible penalty in the absence of GI while a GI length of 12 is required to achieve similar performance in 16QAM C-OFDM. We have also shown that by eliminating the GI, 112-Gbit/s PDM offset-QPSK OFDM can achieve $23 \%$ increase in the net data rate when compared to C-OFDM under the same transmission reach. Consequently, DFT-based offset-QAM OFDM is more suitable than C-OFDM for long-distance fiber transmissions. This work was supported by Science Foundation Ireland under grant number 11/SIRG/I2124.

\section{References}

1. B. Inan et al, "Real-time 93.8-Gb/s polarization multiplexed OFDM transmitter with 1024-point IFFT," Opt. Express, vol. 19, B64, 2011.

2. Q. Zhuge et al, "Dispersion enhanced phase noise effects on reduced guard interval CO-OFDM," Opt. Express, vol. 19, pp. 4472, 2011.

3. J. Zhao et al, "Discrete-Fourier transform based implementation for optical fast OFDM," ECOC'2010, Tu.4.A.3.

4. J. Zhao et al, "Offset-QAM based coherent WDM for spectral efficiency enhancement," Opt. Express, vol. 19, pp. 14617, 2011.

5. S. Randel et al, "Study of multicarrier offset-QAM for spectrally efficient coherent optical communications," ECOC'2011, Th.11.A.1.

6. J. Zhao, "DFT-based offset-QAM OFDM with arbitrary orthogonal waveform generation," ECOC'2013, P.3.10. 